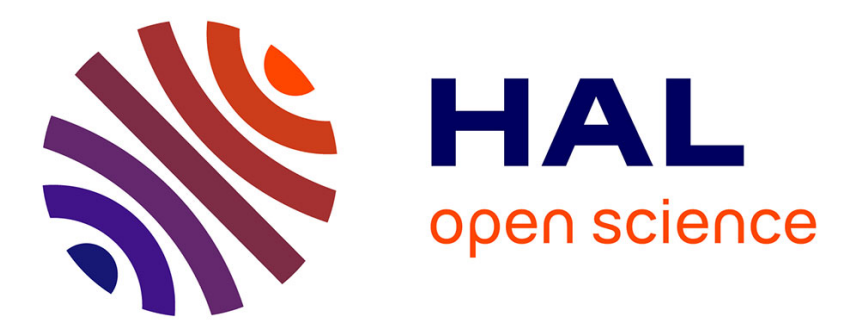

\title{
Complex dynamics of a nonlinear aerospace structure: numerical continuation and normal modes
}

\author{
Ludovic Renson, Jean-Philippe Noël, Gaëtan Kerschen
}

\section{To cite this version:}

Ludovic Renson, Jean-Philippe Noël, Gaëtan Kerschen. Complex dynamics of a nonlinear aerospace structure: numerical continuation and normal modes. Nonlinear Dynamics, 2015, 79 (2), pp.12931309. 10.1007/s11071-014-1743-0 . hal-01635221

\section{HAL Id: hal-01635221 https://hal.science/hal-01635221}

Submitted on 14 Nov 2017

HAL is a multi-disciplinary open access archive for the deposit and dissemination of scientific research documents, whether they are published or not. The documents may come from teaching and research institutions in France or abroad, or from public or private research centers.
L'archive ouverte pluridisciplinaire HAL, est destinée au dépôt et à la diffusion de documents scientifiques de niveau recherche, publiés ou non, émanant des établissements d'enseignement et de recherche français ou étrangers, des laboratoires publics ou privés. 


\title{
Complex dynamics of a nonlinear aerospace structure: numerical continuation and normal modes
}

\author{
L. Renson · J. P. Noël • G. Kerschen
}

\begin{abstract}
This paper investigates the dynamics of a real-life aerospace structure possessing a strongly nonlinear component with multiple mechanical stops. A full-scale finite element model is built for gaining additional insight into the nonlinear dynamics that was observed experimentally, but also for uncovering additional nonlinear phenomena, such as quasiperiodic regimes of motion. Forced/unforced, damped/ undamped numerical simulations are carried out using advanced techniques and theoretical concepts such as numerical continuation and nonlinear normal modes.
\end{abstract}

Keywords Aerospace structure - Piecewise-linear nonlinearities $\cdot$ Numerical continuation $\cdot$ Nonlinear normal modes $\cdot$ Modal interactions

\section{Introduction}

It is widely accepted that virtually all engineering structures are nonlinear, at least in certain regimes of motion. Even if the common industrial practice is to ignore

L. Renson $(\varangle) \cdot$ J. P. Noël · G. Kerschen

Space Structures and Systems Laboratory, Aerospace and Mechanical Engineering Department, University of Liège, Liège, Belgium

e-mail:1.renson@ulg.ac.be

J. P. Noël

e-mail:.jp.noel@ulg.ac.be

G. Kerschen

e-mail: g.kerschen@ulg.ac.be nonlinearity, a recent trend is to exploit them for engineering design, e.g., for vibration absorption and mitigation [1-3]. The last decade witnessed progresses in this direction, and, in particular, in the analysis of nonlinear aerospace structures. Experimental identification of nonlinearity during aircraft and helicopter ground vibration tests was, for instance, performed in references [4-8]. Nonlinearity was also evidenced and identified during spacecraft testing $[9,10]$. However, most of the existing experimental contributions assumed or observed weakly nonlinear behaviors. In parallel, substantial efforts were made to address the numerical modeling of complex, nonlinear aerospace structures (see, e.g., [11,12]). Analysis using advanced numerical continuation techniques was also carried out in [13,14].

Very few studies attempted to numerically analyze and experimentally compare the dynamics of a real-life structure in strongly nonlinear regimes of motion. This is the main contribution of the present paper. The identification of the SmallSat spacecraft, a satellite possessing a nonlinear component with multiple axial and lateral mechanical stops, was achieved in [15] using measurements collected during a typical qualification test campaign. This study revealed that the spacecraft may exhibit complex dynamical phenomena in commonly endured experimental conditions. For instance, jumps, interactions between modes with noncommensurate linear frequencies, force relaxation, and chattering during impacts on the mechanical stops were reported in [15]. Furthermore, several interactions between local and global modes of the structure evidenced energy 
transfers to the payload, which jeopardize its structural integrity and, in turn, the satellite's mission. Understanding and predicting these phenomena are thus of the utmost importance.

This paper builds a full-scale computational model of the satellite for gaining further insight into the observed nonlinear dynamics, but also for uncovering additional nonlinear phenomena not reproduced experimentally. Forced/unforced, damped/undamped numerical simulations are carried out using advanced techniques and theoretical concepts such as numerical continuation $[16,17]$ and nonlinear normal modes $[18,19]$. We note that a formal model updating process could not be achieved during the test campaign. Bringing the predictions of the model in very close quantitative agreement with the experimental results is therefore not the objective of this paper.

The paper is organized as follows. A detailed finite element model of the underlying linear satellite is first built in Sect. 2 and reduced using the Craig-Bampton technique. The model identified experimentally for the nonlinear vibration isolation device is presented and incorporated in the finite element model in Sect. 3. The nonsmooth nonlinearities in the model are regularized for facilitating the ensuing numerical simulations. Sect. 4 provides the numerical evidence of some of the phenomena observed experimentally. A bifurcation analysis then reveals the existence of quasiperiodic regimes of motion. Section 5 carries out nonlinear modal analysis of the SmallSat spacecraft. It discusses in great detail the behavior of several nonlinear modes exhibiting nonlinear modal interactions and energy localization. The conclusions of this study are drawn in Sect. 6.

\section{The SmallSat spacecraft structure}

The SmallSat structure was conceived by EADSAstrium as a low-cost platform for small satellites in low-earth orbits [20]. It is a monocoque tube structure which is $1.2 \mathrm{~m}$ in height and $1 \mathrm{~m}$ in width. It is composed of eight flat faces for equipment mounting purposes, creating an octagon shape, as shown in Fig. 1a. The octagon is manufactured using carbonfiber-reinforced plastic by means of a filament winding process. The structure thickness is $4 \mathrm{~mm}$ with an additional 0.25 -mm-thick skin of Kevlar applied to both the inside and outside surfaces to provide protection against debris. The top floor is an $1-\mathrm{m}^{2}$ sandwich aluminum panel, with 25-mm core and 1-mm skins. The interface between the spacecraft and the launch vehicle is achieved via four aluminum brackets located around cut-outs at the base of the structure. The total mass including the interface brackets is around $64 \mathrm{~kg}$.

The spacecraft structure supports a dummy telescope mounted on a baseplate through a tripod; its mass is around $140 \mathrm{~kg}$. The dummy telescope plate is connected to the SmallSat top floor by three shock attenuators, termed shock attenuation systems for spacecraft and adaptor (SASSAs) [21], whose dynamic behavior may exhibit nonlinearity. Besides, as depicted in Fig. 1b, a support bracket connects to one of the eight walls the so-called wheel elastomer mounting system (WEMS), which is loaded with an 8-kg dummy inertia wheel. The WEMS acts as a mechanical filter, which mitigates high-frequency disturbances coming from the inertia wheel through the presence of a soft elastomeric interface between its mobile part, i.e., the inertia wheel and a supporting metallic cross, and its fixed part, i.e the bracket and by extension the spacecraft. Moreover, the WEMS incorporates eight mechanical stops, covered with a thin layer of elastomer and designed to limit the axial and lateral motions of the inertia wheel during launch, which gives rise to strongly nonlinear dynamical phenomena (cf. Sect. 3).

\subsection{Finite element modeling of the underlying linear satellite}

A finite element model (FEM) of the SmallSat satellite created in the LMS-SAMTECH SAMCEF software is used in the present study to conduct numerical experiments. The model is presented in Fig. 1c, and it comprises about 150,000 degrees of freedom (DOFs). It idealizes the composite tube structure using orthotropic shell elements. The top floor, the bracket, and the wheel support are also modeled using shell elements. Boundary conditions are enforced at the base of the satellite through four clamped nodes. Proportional damping using the parameters provided by EADS-Astrium is also introduced in the model.

The typical frequency range of interest for spacecraft testing is between 5 and $100 \mathrm{~Hz}$. Within this frequency interval, the model comprises 18 linear modes that can be classified into three groups of six modes, as listed in Table 1. The first group, between 8 and 


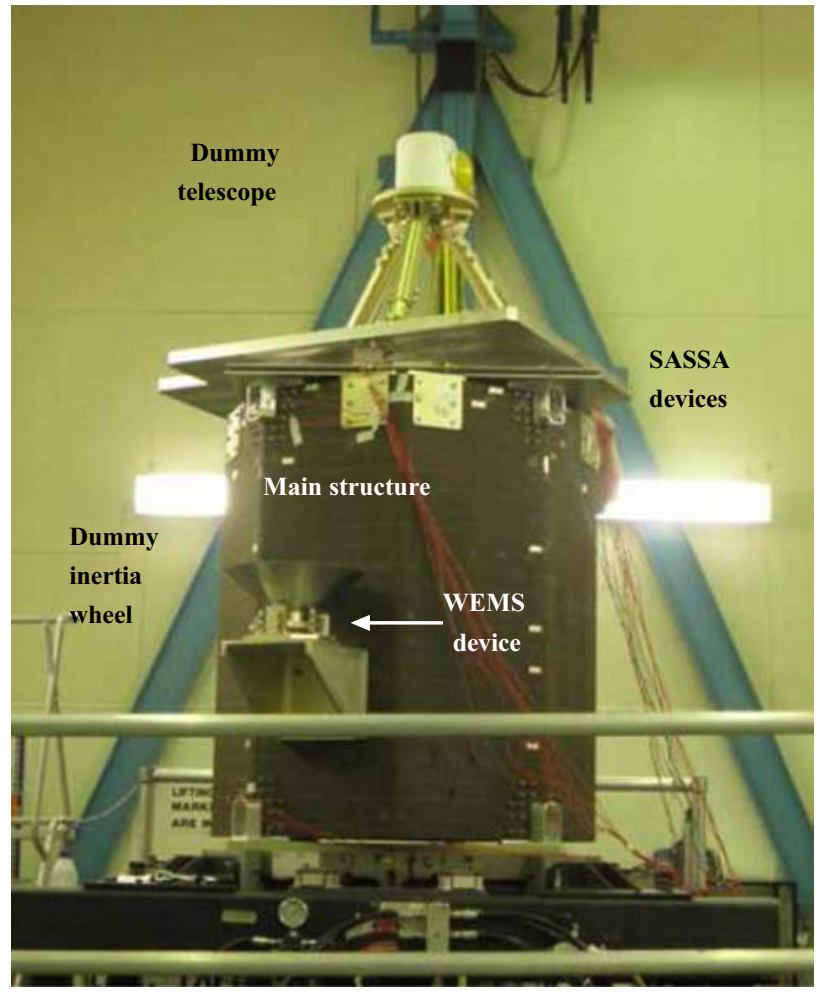

(a)
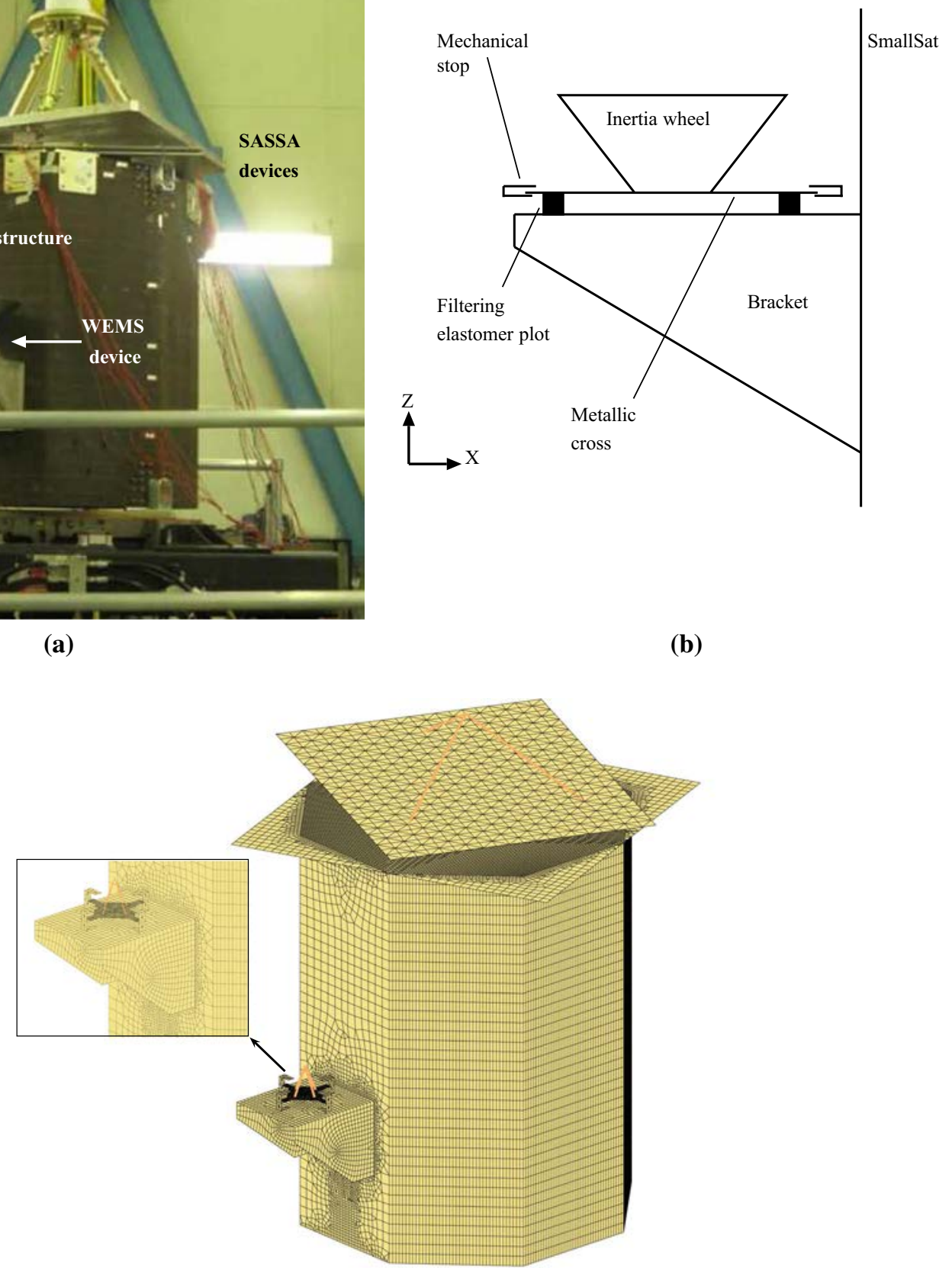

(b)

Fig. 1 SmallSat spacecraft equipped with an inertia wheel supported by the WEMS and a dummy telescope connected to the main structure by the SASSA isolators. a Photograph; b schematic of the nonlinear vibration isolation device; $\mathbf{c}$ finite element model 
Table 1 Comparison between numerical and experimental natural frequencies

\begin{tabular}{lcc}
\hline Mode \# & Model freq. (Hz) & Experimental freq. (Hz) \\
\hline 1 & 8.06 & 8.19 \\
2 & 9.14 & - \\
3 & 20.44 & - \\
4 & 21.59 & - \\
5 & 22.05 & 20.18 \\
6 & 28.75 & 22.45 \\
7 & 32.49 & - \\
8 & 34.78 & 34.30 \\
9 & 39.07 & - \\
10 & 40.78 & 43.16 \\
11 & 45.78 & 45.99 \\
12 & 57.76 & 55.71 \\
13 & 68.99 & 64.60 \\
14 & 75.14 & - \\
15 & 79.82 & - \\
16 & 83.36 & - \\
17 & 89.01 & 88.24 \\
18 & 95.30 & - \\
\hline
\end{tabular}

A dash means that the corresponding mode could not be identified during the test campaign

$29 \mathrm{~Hz}$, shows local WEMS motions. The corresponding modal shapes are depicted in Fig. 2. Modes 1 and 2 show a concave trajectory of the WEMS about $Y$ and $X$ axes, respectively. Modes 3 and 5 correspond to a convex trajectory of the WEMS about $Y$ and $X$ axes, respectively. The fourth mode presents an in-plane rotation and extension of the WEMS cross. Only mode 6 combines a significant bracket deflection with a vertical WEMS motion. The second group, between 32 and $58 \mathrm{~Hz}$, is composed of local SASSA modes including global deformation of the main structure. The last group comprises modes with local deformation of the main structure panels often combined with bracket deformation.

Low-level random data acquired during the test campaign were used in [15] to extract the modal parameters of the underlying linear satellite. As stressed in the introductory section, a formal model updating process could not be achieved during the test campaign. Nonetheless, the good agreement in Table 1 between the natural frequencies predicted by the FEM and those identified experimentally together with the correct mode ordering confirm that the model should have satisfactory predictive capabilities.

\subsection{Reduced-order modeling}

Because the WEMS nonlinearities are spatially localized, condensation of the linear FEM can be effectively achieved using the Craig-Bampton reduction technique [22]. This leads to a substantial decrease in the computational burden without degrading the accuracy of the numerical simulations in the frequency range of interest. The Craig-Bampton method expresses the complete set of initial DOFs in terms of retained DOFs and internal vibration modes of the structure clamped on the retained nodes. To introduce the WEMS nonlinearities, the reduced-order model (ROM) is constructed by keeping one node on both sides of the lateral and axial mechanical stops. In total, eight nodes of the initial FEM possessing 3 DOFs each and 10 internal modes of vibration are kept; this reduced model possesses 34 DOFs and is termed ROM810. For local excitation of the WEMS, a second ROM, termed ROM910, is created with an additional node on the metallic cross.

The ROM accuracy is assessed by comparing its modal parameters with those of the original full-scale model. The deviation between the mode shapes is determined using the modal assurance criterion (MAC). MAC value ranges from 0 in the absence of correlation to 1 for a complete correspondence. The frequency deviations as well as the MAC of ROM810 are displayed in Fig. 3. We observe a very good correlation for the first 18 modes which cover the frequency range of interest. We, however, note that, below 10 internal modes, the ROM cannot simultaneously capture the first group of WEMS local modes and the second and third groups.

\section{Modeling of the WEMS nonlinearities}

Figure 4a presents a simplified, yet relevant, modeling of the WEMS where the inertia wheel, owing to its important rigidity, is seen as a point mass. The four nonlinear connections (NCs) between the WEMS mobile and fixed parts are labeled NC1-4, respectively.

The WEMS nonlinearities are the only nonlinear components introduced in the model. They were 


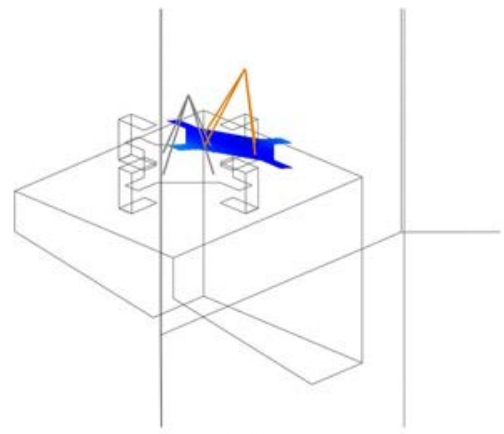

(1)

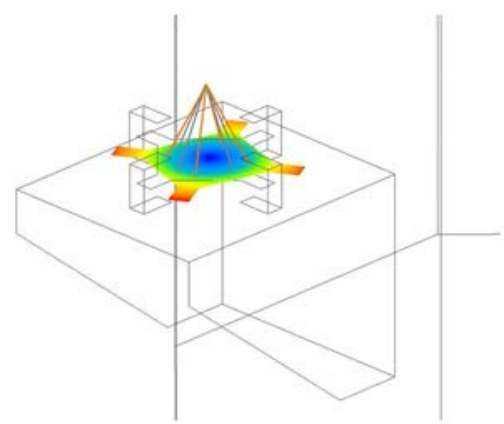

(4)

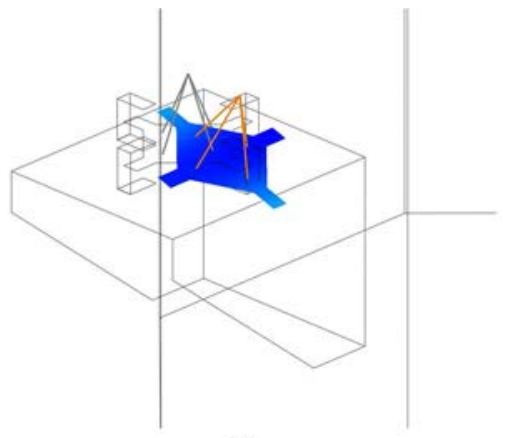

(2)

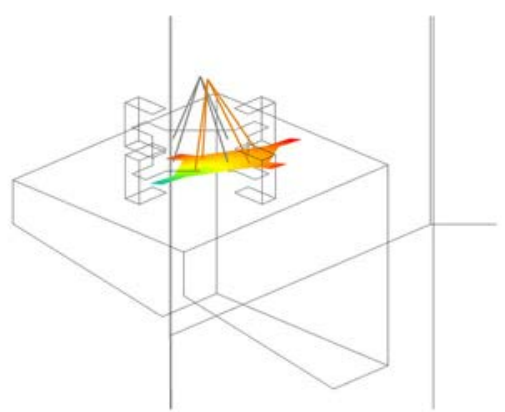

(5)

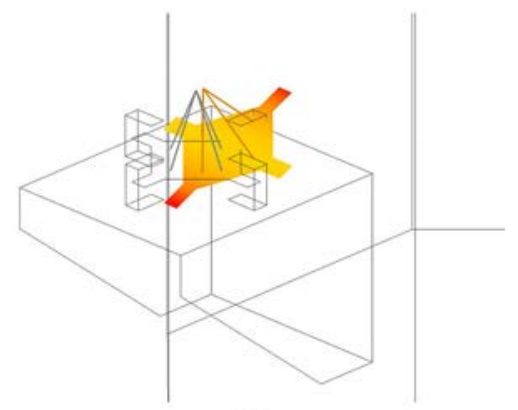

(3)

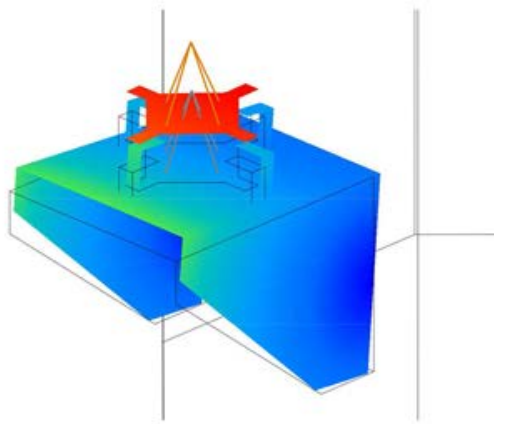

(6)

Fig. 2 Close-up of the six first LNMs (local WEMS motion). (1-6) for LNM 1-LNM 6, respectively

Fig. 3 Relative error on frequencies and MAC between the ROM810 and the original model

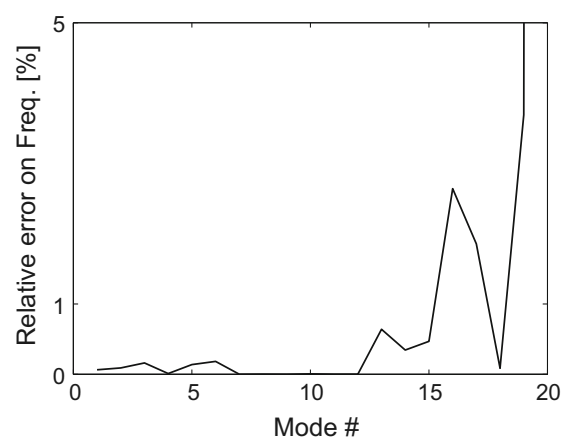

(a)

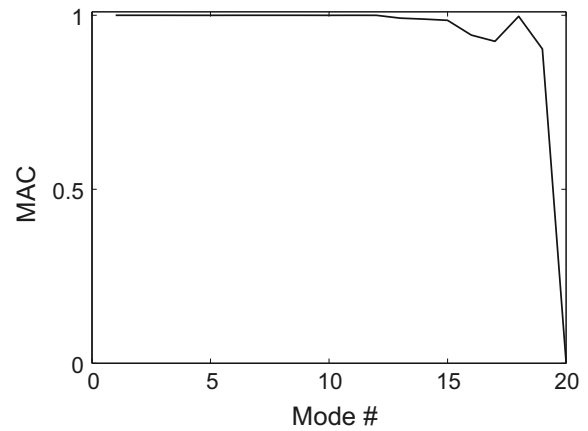

(b) accurately identified in [15] using measured data from swept-sine base excitations at different amplitude levels. For instance, the stiffness curve characterizing $\mathrm{NC} 1$, identified using the restoring force surface method (RFS) [23], is depicted in Fig. 4b. It turns out from this figure that the WEMS modeling should account for combined nonsmooth and gravity-induced asymmetric effects. This leads us to select a trilinear model $k_{-}, k$, and $k_{+}$with dissimilar clearances $a_{-}$and $a_{+}$for the axial nonlinearities. This complex model is in better agreement with reality than the symmetric model considered in earlier investigations [24,25]. For the lateral nonlinearities, a bilinear model $k_{ \pm}$and $k$ suffices, because there is only one clearance $a_{ \pm}$per connection. A curve-fitting process, represented by the red curve in Fig. 4b, provides the unknown parameters for our piecewise-linear model (see Table 2). For confidentiality, stiffness coefficients and clearances are given through adimensionalised quantities. Localized damping terms given by EADS-Astrium were also included in the FEM to account for the dissipation of the elastomer plots. 


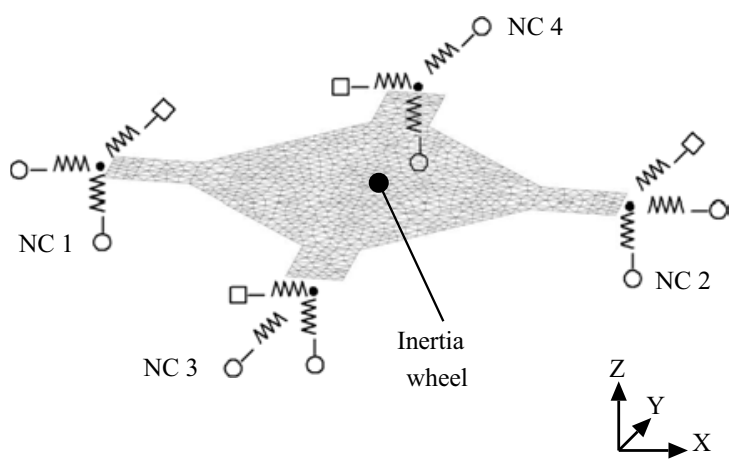

(a)

Fig. 4 WEMS. a Simplified modeling of the WEMS mobile part considering the inertia wheel as a point mass. The linear and nonlinear connections between the WEMS mobile and fixed parts are signaled by open squares and open circles, respectively.

Table 2 Parameters of the WEMS nonlinear connections (adimensionalised for confidentiality)

\begin{tabular}{lllll}
\hline Stiffness & NC1 & NC2 & NC4 & NC3 \\
\hline Axial $k_{Z}$ & 8.30 & 9.21 & 9.18 & 10.03 \\
Lateral $k_{X}$ & 1.31 & 1.31 & 0.69 & 0.69 \\
Lateral $k_{Y}$ & 0.69 & 0.69 & 0.69 & 0.69 \\
Axial $k_{+, Z}$ & 79.40 & 88.41 & 79.40 & 88.41 \\
Axial $k_{-, Z}$ & 118.07 & 116.73 & 118.07 & 116.73 \\
Lateral $k_{ \pm, X Y}$ & 40 & 40 & 40 & 40 \\
Clearance & & & & \\
Axial $a_{+, Z}$ & 1.55 & 1.62 & 1.59 & 1.59 \\
Axial $a_{-, Z}$ & 1.01 & 0.84 & 0.93 & 0.93 \\
Lateral $a_{ \pm, X Y}$ & 2 & 2 & 2 & 2 \\
\hline
\end{tabular}

Finally, for facilitating the numerical investigations in the forthcoming sections, the continuity of the first derivative of the different restoring forces of the WEMS is enforced using regularization. This approach is also motivated by the stiffness curve in Fig. 4b, which reveals that the actual structural behavior is smoother than a piecewise-linear law. A local regularization using Hermite polynomials in the interval $[a-\Delta, a+\Delta]$ is considered where $a$ and $2 \Delta$ are the clearance and the size of the regularization interval, respectively. The nominal interval considered throughout the paper is equal to $5 \%$ of the clearance size. As illustrated in Fig. 5, the main advantage of this strat-

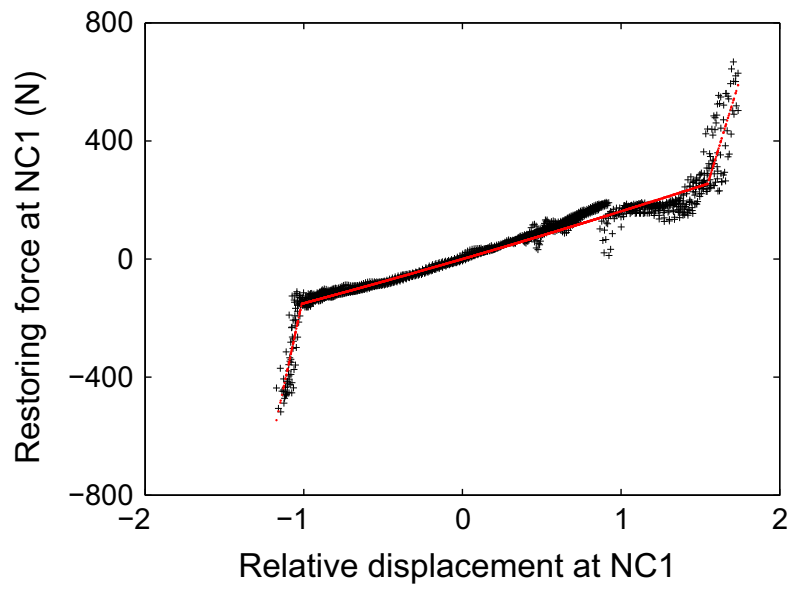

(b)

b Experimental stiffness curve of NC1 constructed using the RFS method (in black) and fitted with a trilinear model (in red). (Color figure online)

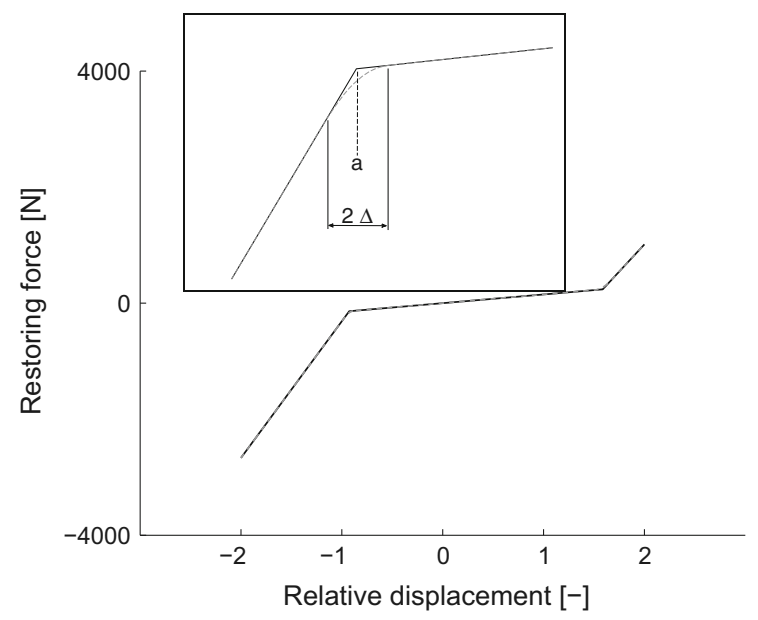

Fig. 5 Axial WEMS nonlinearity modeling. True piecewiselinear (lines) and regularized (dash lines) restoring forces

egy is that it preserves the purely linear behavior of the restoring force outside the regularization interval.

The mathematical description of the nonlinear force is given by

$$
\begin{aligned}
f_{\mathrm{nl}}(x)= & \left\{\begin{array}{l}
\operatorname{sign}(x)\left(k a_{+}+k_{+}\left(x-a_{+}\right)\right) \\
p_{+}(t(x)) \\
k x \\
p_{-}(t(x)) \\
\operatorname{sign}(x)\left(k a_{-}+k_{-}\left(x-a_{-}\right)\right)
\end{array}\right. \\
& x \geq a_{+}+\Delta_{+}
\end{aligned}
$$




$$
\begin{aligned}
& a_{+}+\Delta_{+}>x>a_{+}-\Delta_{+} \\
& a_{+}-\Delta_{+} \geq x \geq-\left(a_{-}-\Delta_{-}\right) \\
& -\left(a_{-}-\Delta_{-}\right)>x>-\left(a_{-}+\Delta_{-}\right) \\
& x \leq-\left(a_{-}+\Delta_{-}\right)
\end{aligned}
$$

where $x$ is the relative displacement between the two DOFs defining the NC. The parameters $a_{ \pm}, k_{ \pm}$, and $\Delta_{ \pm}$are positive scalars. The Hermite polynomials are defined as

$$
\begin{aligned}
p_{ \pm}(t)= & h_{00}(t) p_{k}+h_{10}(t)\left(x_{k+1}-x_{k}\right) m_{k} \\
& +h_{01}(t) p_{k+1}+h_{11}(t)\left(x_{k+1}-x_{k}\right) m_{k+1}
\end{aligned}
$$

where $p_{k}$ and $p_{k+1}$ are the values of the restoring force at points $x_{k}=\operatorname{sign}(x)(a-\Delta)$ and $x_{k+1}=$ $\operatorname{sign}(x)(a+\Delta)$, respectively. $m_{k}$ and $m_{k+1}$ are the values of the restoring force derivative at the same $x_{k}$ and $x_{k+1}$ points; they correspond to the stiffness coefficients $k_{1}$ and $k_{ \pm}$, respectively. The local scaled abscissa is

$t(x)=\frac{x-x_{k}}{x_{k+1}-x_{k}}$.

The $h_{i j}(t)$ functions are given by

$h_{00}(t)=2 t^{3}-3 t^{2}+1$,

$h_{10}(t)=t^{3}-2 t^{2}+t$,

$h_{01}(t)=-2 t^{3}+3 t^{2}$,

$h_{11}(t)=t^{3}-t^{2}$.

\section{Direct numerical integration and numerical continuation}

The reduced model ROM910 including the WEMS nonlinearities is now used to conduct numerical simulations. To this end, a swept-sine excitation with a sweep rate of 2 octaves per minute in the $5-90 \mathrm{~Hz}$ range is applied to the inertial wheel in the axial direction. The excitation amplitude is $140 \mathrm{~N}$. Direct numerical integration of the equations of motion is carried out using Newmark's algorithm. Figure 6a represents the axial displacement of the NC2 as a function of the excitation frequency. A main resonance peak which corresponds to mode 6 is located around $33 \mathrm{~Hz}$. Because the corresponding linear natural frequency is $28.75 \mathrm{~Hz}$, this nonlinear mode undergoes a substantial increase in frequency due to the frequency-energy dependence of nonlinear oscillations. The asymmetry, nonsmoothness, and skewness of the displacement envelope in the vicinity of the resonance peak are additional manifestations of the WEMS nonlinearities. A sudden transition from large to small amplitudes of vibration, referred to as a jump phenomenon, is also observed. After the jump, the satellite response quickly stabilizes to a lowamplitude response with almost no beating phenomenon; it is a sign of the presence of strong damping. The wavelet transform of the displacement signal is plotted in Fig. 6b. The amplitude of the wavelet transform is represented in logarithmic scale ranging from blue (low amplitude) to red (high amplitude). The presence of wideband frequency components around $30 \mathrm{~Hz}$ confirms the activation of nonsmooth nonlinearities in the neighborhood of the resonance. Their disappearance closely coincides with the jump phenomenon. Even harmonics in the wavelet transform are generated by the asymmetric modeling of the WEMS. The results in Fig. 6 therefore present a very good qualitative concordance with those observed experimentally for mode 1 in [15].

In order to gain further insight into the experimental results, an algorithm for numerical continuation is employed to compute the system response to a $140 \mathrm{~N}$
Fig. 6 NC2-Z response to swept-sine excitation. a Displacement; b wavelet spectrum

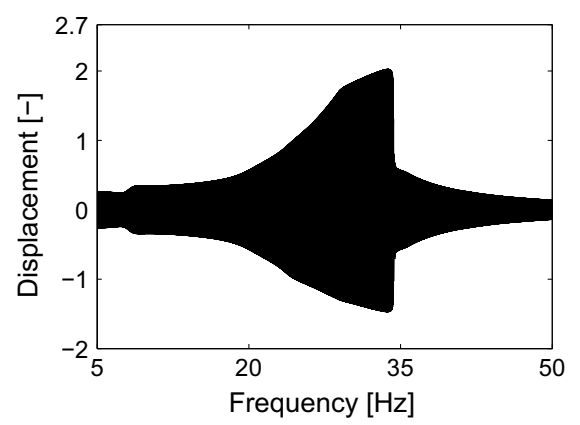

(a)

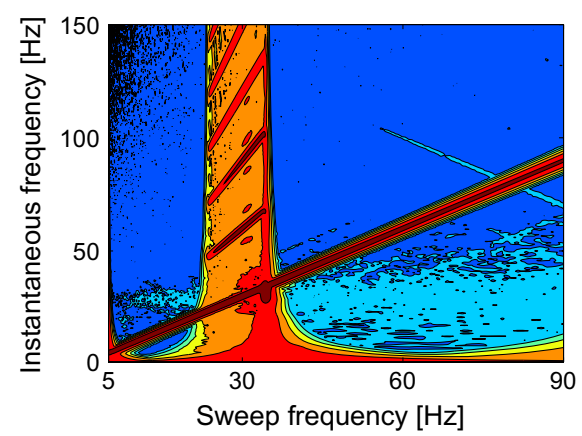

(b) 


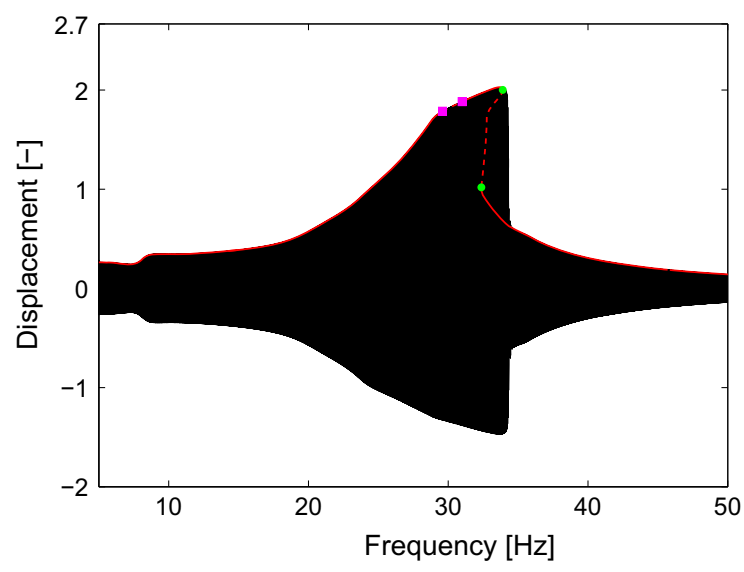

Fig. 7 SmallSat displacement response at NC2-Z. The responses to swept-sine (direct simulations, 2 octaves per minute) and stepped-sine (numerical continuation) excitations are depicted in black and red, respectively. The red solid and dashed lines correspond to stable and unstable periodic solutions, respectively. Limit point and Neimark-Sacker bifurcations are pictured with green circle and magenta squares, respectively. (Color figure online)

stepped-sine forcing. A shooting technique for computing isolated periodic solutions is combined with pseudoarclength continuation for tracking the evolution of the periodic solutions for increasing excitation frequencies [26]. Bifurcation analysis is performed using test functions based on the monodromy matrix [27]. The results of numerical continuation are shown in Fig. 7. They are superimposed on the time series

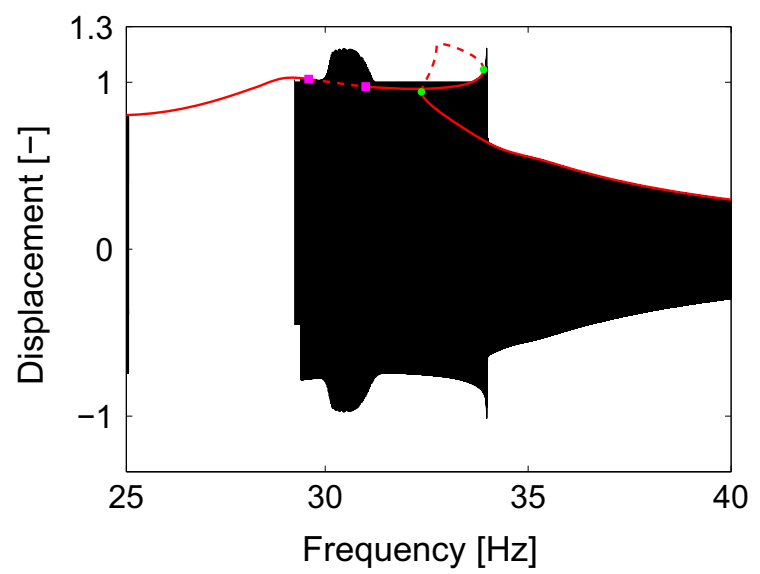

(a)

Fig. 8 Evidence of QP motion. a Displacement at NC1-Z and b close-up of the transition from periodic to QP motion. The responses to swept-sine (direct simulations) and stepped-sine (numerical continuation) excitations are depicted in black and of Fig. 6a and provide an accurate estimation of the displacement envelope. Two limit point (LP) bifurcations give rise to a change in stability of the periodic solutions. The upper LP coincides with the jump phenomenon and explains why there is a sudden transition between two stable attractors characterized by large and small amplitudes, respectively. This is a classical theoretical result in the literature, often illustrated using single-DOF oscillators, but these simulations show that it can also be observed during testing of real-life engineering structures.

Another objective of this section is to uncover nonlinear dynamical phenomena not encountered experimentally in [15]. Interestingly, the numerical continuation process highlights that the periodic solutions undergo two additional Neimark-Sacker bifurcations [27]. In addition to changing the stability of the periodic solutions, this type of bifurcation produces an emerging torus on which quasiperiodic $(\mathrm{QP})$ motion may occur. Such a motion was not present in the time series of Fig. 6a. However, numerical simulations for slower sweep rates, e.g., for a linear sweep rate of $10 \mathrm{~Hz}$ per minute as in Fig. 8, show that QP motion can be observed. The envelope of the displacement signal increases rapidly after the first Neimark-Sacker bifurcation, and periodic motion degenerates into QP motion. After the second bifurcation, the QP motion is transformed into periodic motion, and the envelope decreases rapidly. There is a small delay between the

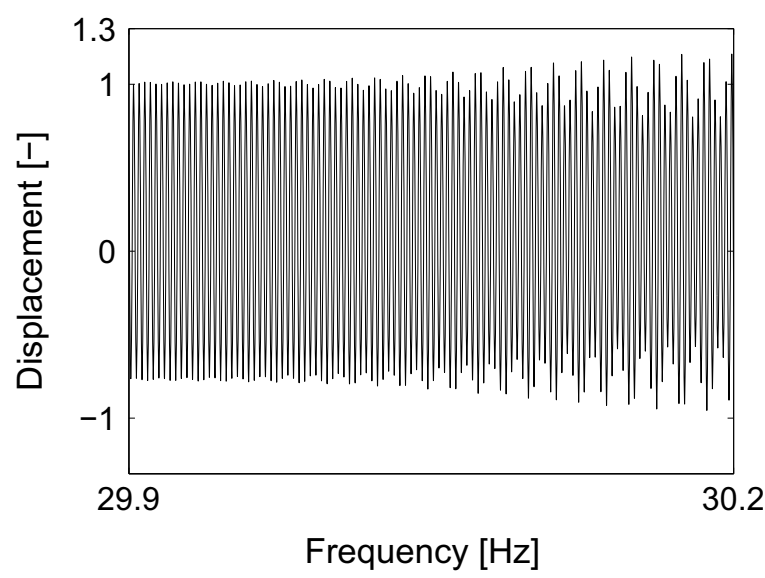

(b)

red, respectively. Limit point and Neimark-Sacker bifurcations are pictured with green circle and magenta squares, respectively. (Color figure online) 
first (second) bifurcation and the onset (disappearance) of QP motion; this delay is to be attributed to the transient character of the swept-sine excitation. Figure 8b shows that the time series associated with QP motion also exhibits asymmetric behavior with dominant positive displacements. Overall, this complex, inherently nonlinear dynamical behavior is of important practical significance, because, as displayed in Fig. 8a, the vibration amplitude associated with QP motion is as large as the amplitude close to the main resonance of the system.

\section{Nonlinear modal analysis of the SmallSat spacecraft}

In the previous section, a nonconservative FEM was utilized to further investigate the nonlinear phenomena observed during the testing campaign of the SmallSat spacecraft. Because the damped dynamics can also be interpreted based on the topological structure and the bifurcations of the nonlinear normal modes (NNMs) of the underlying conservative system [28], a detailed nonlinear modal analysis is carried out herein.

An extension of Rosenberg's definition is considered, i.e., an NNM is defined as a (nonnecessarily synchronous) periodic motion of the unforced, conservative system. The algorithm proposed in [29], which combines shooting and pseudoarclength continuation, is applied to the ROM810 model for NNM computation. Due to the frequency-energy dependence of nonlinear oscillations, NNMs are depicted in a frequencyenergy plot (FEP). An NNM is represented by a point in the FEP, drawn at a frequency corresponding to the

Fig. 9 Mode 9. a Linear modal shape; $\mathbf{b}$ frequency-energy plot minimal period of the periodic motion, and at an energy equal to the conserved total energy during the motion. A branch depicted by a solid line represents the complete frequency-energy dependence of the considered mode.

Unlike a previous application of the NNM theory to a full-scale aircraft [30], an interesting feature of the satellite is that almost every mode of the underlying linear system in the $[0-100] \mathrm{Hz}$ range involves motion of the nonlinear component. The exception is mode 9 for which the WEMS remains quiescent, as shown in Fig. 9a. This is confirmed by the constant natural frequency of NNM9 in the FEP of Fig. 9b. Modes 1, 5, 6 , and 7 were found to exhibit particularly interesting dynamics and are described in this section.

The first linear normal mode (LNM1) corresponds to a local motion involving the WEMS. Its nonlinear counterpart is pictured in Fig. 10. The FEP of NNM1 is formed by one main backbone to which one "tongue" is attached. At low energies, no mechanical stop is activated, and the NNM frequency remains identical to the natural frequency of LNM1. The corresponding modal shape is also identical to that of LNM1. Beyond a certain energy threshold, the relative displacements along $\mathrm{X}$ of nonlinear connections $\mathrm{NC} 1$ and $\mathrm{NC} 2$ enter into the regularization area of the piecewise-linear restoring forces. The NNM frequency rapidly increases due to the large difference between the stiffnesses of the elastomer plots and of the mechanical stops. When progressing along the backbone, harmonic components of the fundamental NNM oscillation frequency are created by the WEMS nonlinearities. Once one of these harmonics has a frequency close to the oscillation frequency of another NNM, a dynamic coupling between the two modes exists, and a tongue of internal resonance

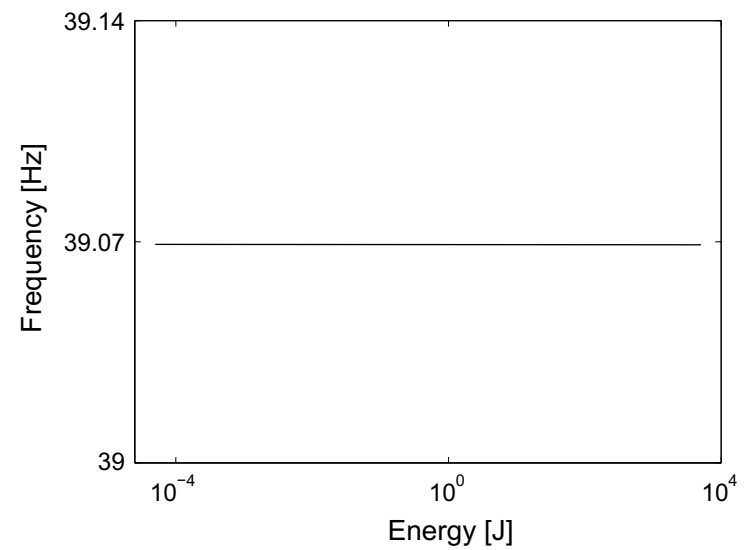

(b) 
Fig. 10 FEP of the first NNM with different modal shapes inset

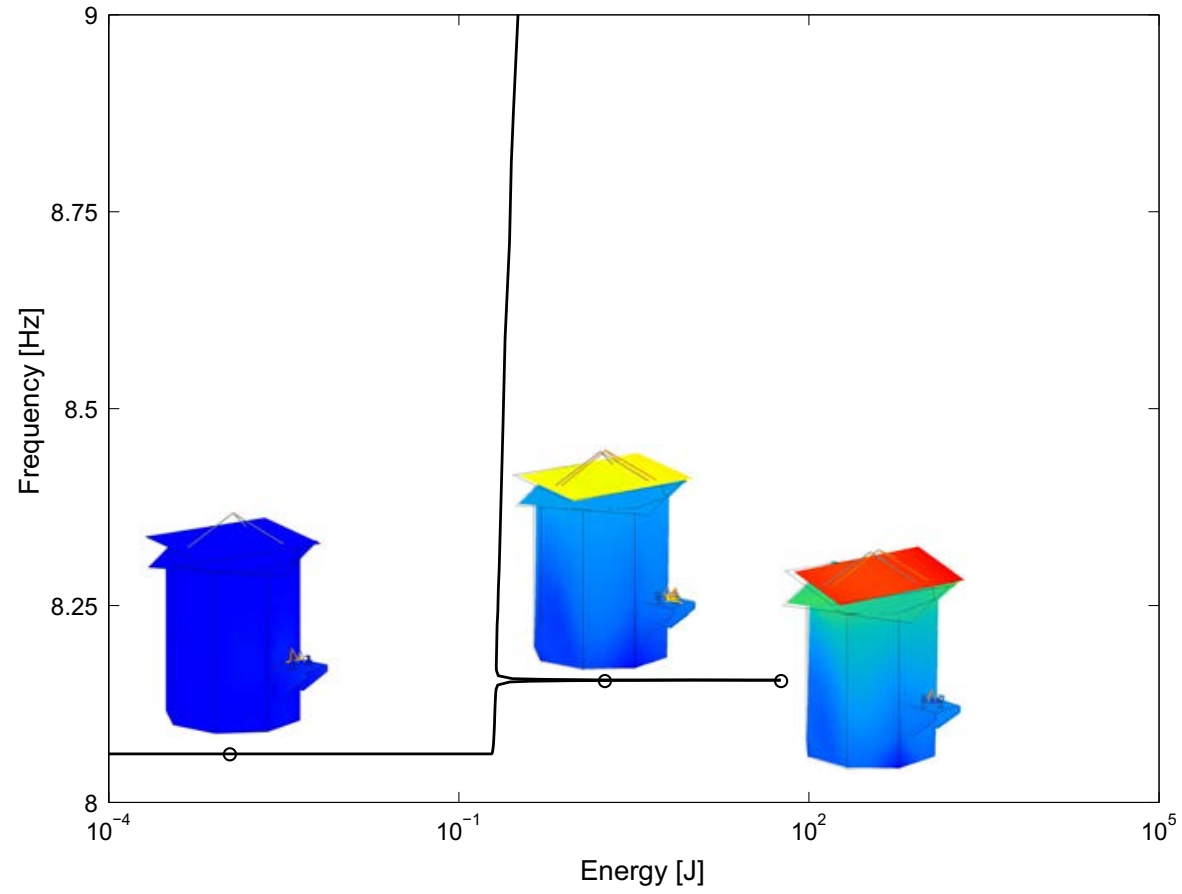

is produced. This is precisely what happens for the 5:1 internal resonance in Fig. 10. As energy increases along this branch, the fifth harmonic becomes more important than the fundamental frequency. The modal shape located around the middle of the branch is a mixing between NNM1 and NNM10; it is a purely nonlinear mode with no linear counterpart. At the extremity, the sole fifth harmonic remains, which completes the transition to NNM10. Such internal resonances between NNMs were previously reported in the literature, see, e.g., [28,31], also in the case of a two-degreeof-freedom vibro-impact system [32] and a full-scale aircraft [30]. They are therefore not further described herein. However, it is interesting to note that, due to nonlinearities, the excitation of a local mode can trigger the excitation of a more global mode involving instrument panel motion. This latter mode is characterized by a much larger modal mass and can potentially jeopardize structural integrity during launch.

Figure 11 presents the FEP of the fifth spacecraft NNM. The same findings as for NNM1 can be drawn from this FEP, namely a sudden increase in NNM frequency once mechanical stops are activated and the presence of internal resonances. One branch of 2:1 internal resonance with NNM11 and two 4:1 branches with NNM17 and 18 are generated due to the asymmet- ric modeling of the WEMS. Branches involving even harmonics were also observed for a system with cubic nonlinearity in [28], but, due to the symmetry of the restoring force, they were created through symmetrybreaking bifurcations. The existence of branches 15:1 and 120:1 is questionable, because the corresponding oscillation frequencies are outside the range of validity of ROM810.

What is particularly interesting with NNM5 is that its 2:1 interaction with NNM11 was observed experimentally in [15]. The experimental evidence is presented in the wavelet transform of Fig. 12. At the NC4-Y sensor, the only visible frequency component is around $45-46 \mathrm{~Hz}$ despite the fact that the excitation frequency is twice smaller, a clear sign of an energy transfer between modes. An important remark is that it is not the experimental mode with a linear frequency of $22.45 \mathrm{~Hz}$ which is involved in the interaction, but rather the experimental mode with a linear frequency of $20.18 \mathrm{~Hz}$ (see Table 1). Due to the hardening effect of the WEMS, the frequency of this latter mode increases up to $22.5-23 \mathrm{~Hz}$ in Fig. 12, which, in turn, triggers the excitation of the experimental mode possessing a linear frequency of $45.99 \mathrm{~Hz}$. To the best of our knowledge, this is the first time that such an agreement between predictions and experiments is reported for an interac- 
Fig. 11 FEP of the fifth NNM with different modal shapes inset

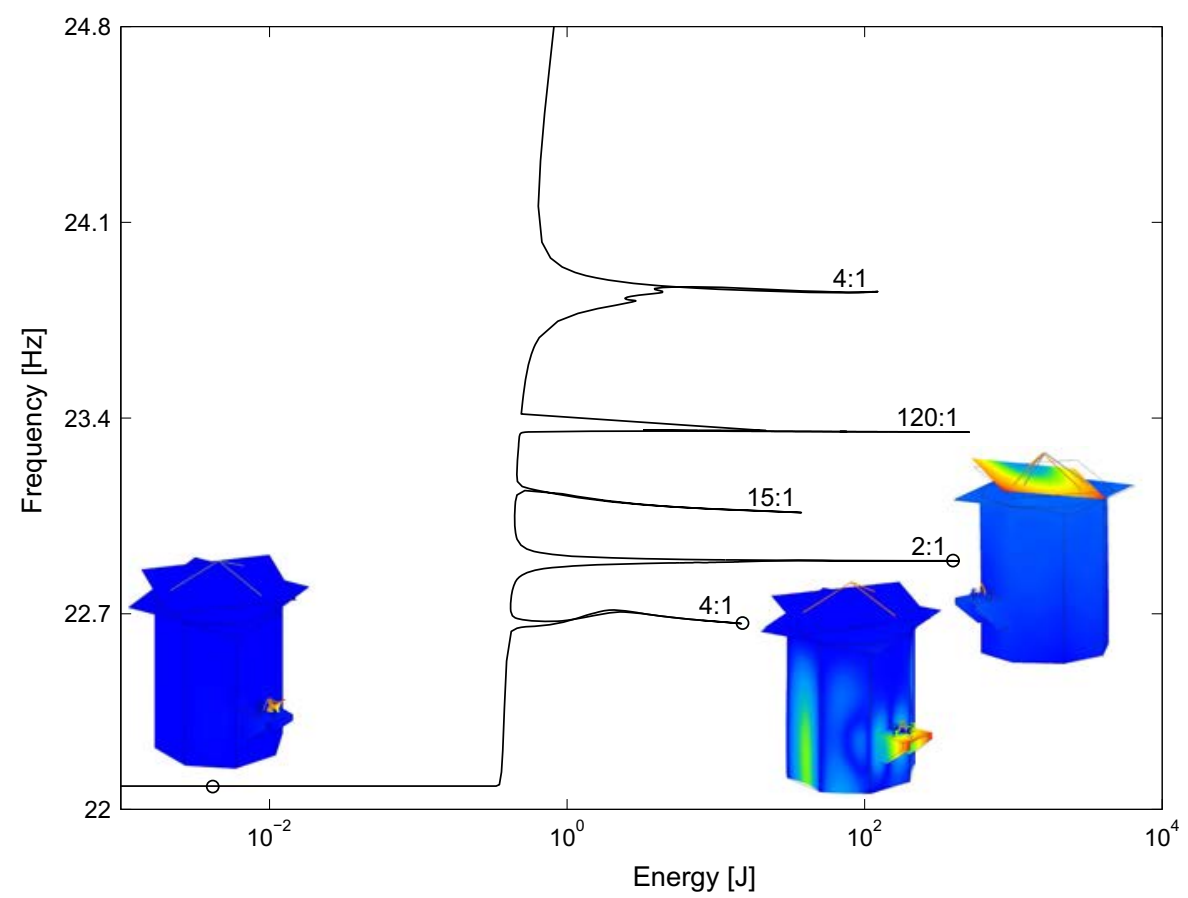

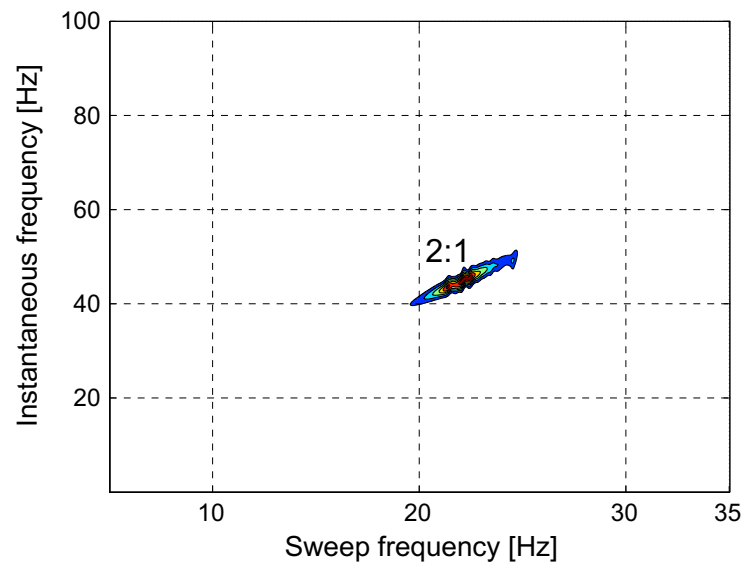

Fig. 12 Wavelet transform of the experimental time series measured at NC4-Y (swept-sine excitation, $1 \mathrm{~g}$ ), [15]

tion between two modes of a real-life structure with noncommensurate linear natural frequencies.

A third local mode of the WEMS, NNM6, is presented in Fig. 13. As for NNM5, there is a 2:1 modal interaction during which NNM6 interacts with NNM12 corresponding to an axial motion of the instrument supporting panel. Numerical evidence of this interaction is provided by analyzing the response at the instrument panel to swept-sine excitation in Fig. 14a. Damping is included in the numerical simulation. For a forcing amplitude of $20 \mathrm{~N}$, the satellite presents several resonance peaks at frequencies equal to the linear natural frequencies (see Table 1). For a forcing amplitude of $80 \mathrm{~N}$, an additional resonance peak corresponding to an excitation frequency of $29 \mathrm{~Hz}$ can be observed. The presence of this resonance cannot be predicted by a linear analysis, because there is no linear mode possessing instrument panel motion below $32 \mathrm{~Hz}$. It is therefore a nonlinear resonance during which the second harmonic of NNM6 characterized by a frequency close to $58 \mathrm{~Hz}$ excites NNM12. This, in turn, produces a large response at the instrument panel when the excitation frequency is in the vicinity of NNM6. Interestingly, this nonlinear resonance has an acceleration twice as large as the acceleration corresponding to the linear resonance of the panel at $58 \mathrm{~Hz}$. Further experimental evidence of the modal interaction is shown in the wavelet transform measured at $\mathrm{NC} 4$ in Fig. 14b [15]. The excitation frequency, denoted by fund., is accompanied with higher harmonic components of comparable amplitudes. Specifically, a second harmonic ranging from 55 to $60 \mathrm{~Hz}$ is visible when the excitation frequency approaches $30 \mathrm{~Hz}$. There is no identified linear mode just below $30 \mathrm{~Hz}$, but, due to the hardening effect of the WEMS, the linear frequency of $22.45 \mathrm{~Hz}$ increases substantially during nonlinear regimes of motion. The second harmonic then 
Fig. 13 FEP of the sixth NNM with different modal shapes inset

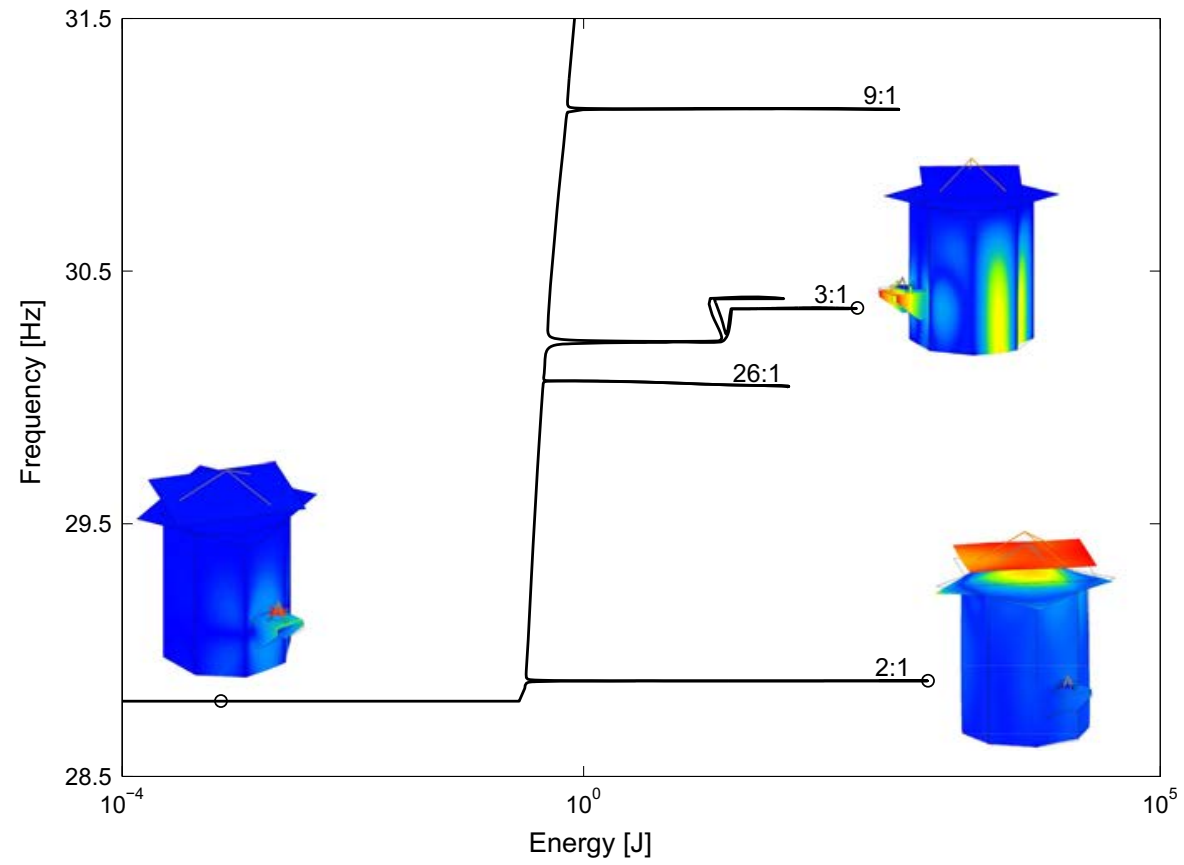

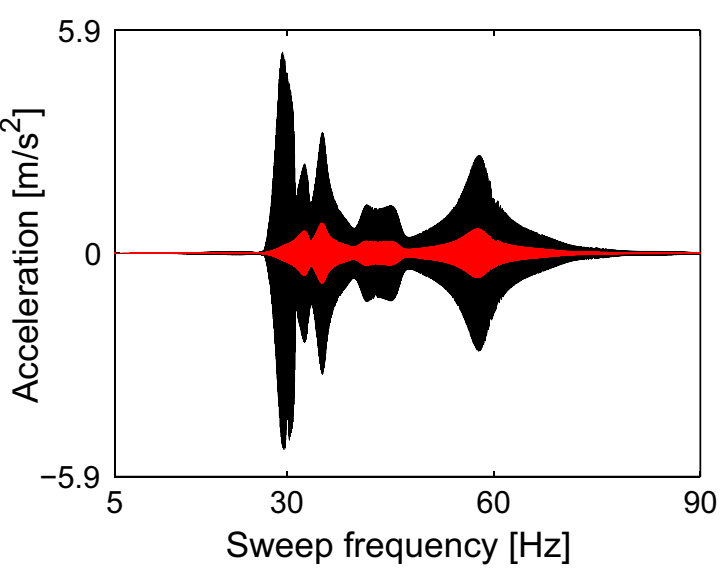

(a)

Fig. 14 Numerical and experimental evidence of the 2:1 modal interaction between NNM6 and NNM12. a Acceleration at the instrument panel for a swept-sine excitation of $20 \mathrm{~N}$ (red) and

excites the experimental mode with a linear frequency of $55.71 \mathrm{~Hz}$.

The 3:1 modal interaction presents a nonconventional topology compared to the other branches in the FEP of Fig. 13. A close-up of the branch is shown in Fig. 15 together with the backbone of NNM17 repre-

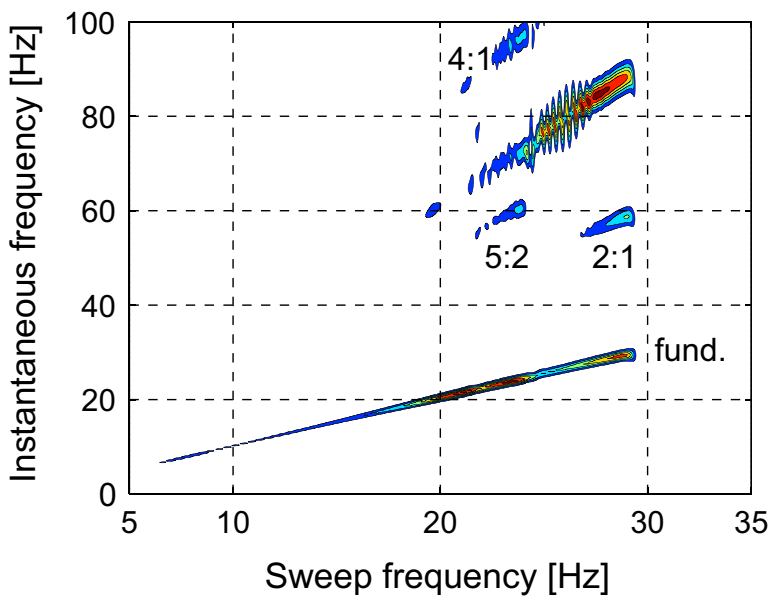

(b)

$80 \mathrm{~N}$ (black) amplitude (direct numerical simulation); b wavelet transform of the relative displacement at NC4-Z (measured during the testing campaign). (Color figure online)

sented at the third of its fundamental frequency. ${ }^{1}$ The reason for this complex topology is that the dynamics has to evolve from NNM6, a mode with a predominant axial motion between the WEMS and the bracket activating a unique axial nonlinear connection, NC2-Z, to NNM17, a mode with lateral motion of the

$\overline{1}$ This is relevant, because a periodic solution of period $T$ is periodic with period $3 T$. 
Table 3 Activation of the nonlinear connections on the 3:1 interaction between NNM6 and NNM17

A zero value indicates that the mechanical stop is not activated. A value greater than one implies that the regularization interval is crossed. + and correspond to positive and negative relative displacements, respectively

\begin{tabular}{llllll}
\hline & $\mathrm{NC} 2-\mathrm{Z}(+,-)$ & $\mathrm{NC} 3-\mathrm{Z}(+,-)$ & $\mathrm{NC} 4-\mathrm{Z}(+,-)$ & $\mathrm{NC} 3-\mathrm{Y}(+,-)$ & $\mathrm{NC4}-\mathrm{Y}(+,-)$ \\
\hline $\mathrm{A}$ & $(0.2 .2)$ & $(0,0)$ & $(0,0)$ & $(0,0)$ & $(0,0)$ \\
$\mathrm{B}$ & $(0,2.2)$ & $(0,0)$ & $(0,0)$ & $(0,0)$ & $(0,0)$ \\
$\mathrm{C}$ & $(0,2.2)$ & $(0,0.07)$ & $(0,0)$ & $(0,0)$ & $(0,0)$ \\
$\mathrm{D}$ & $(0,2.3)$ & $(0,0.19)$ & $(0,0)$ & $(1.1,1.1)$ & $(1.1,1.1)$ \\
E & $(0,0)$ & $(0,0)$ & $(0,2.9)$ & $(3.4,3.6)$ & $(3.4,3.4)$ \\
F & $(0,0)$ & $(0,0)$ & $(0,0)$ & $(3,3)$ & $(3,3)$ \\
G & $(0,0)$ & $(0,0)$ & $(0,0)$ & $(4,4)$ & $(4,4)$ \\
H & $(0,0)$ & $(0,0)$ & $(0,2.7)$ & $(3.5,3.8)$ & $(3.7,3.7)$ \\
$\mathrm{I}$ & $(0,2.1)$ & $(0,0)$ & $(0,1.0)$ & $(0,0)$ & $(0,0)$ \\
J & $(0,2.3)$ & $(0,0)$ & $(0,0)$ & $(0,0)$ & $(0,0)$ \\
\hline
\end{tabular}

bracket activating two other nonlinear connections in the lateral direction, NC3-Y and NC4-Y. To understand this progression, the motion of the center of gravity of the WEMS cross is displayed in Fig. 15. Clearly, the WEMS motion takes place in the XZ plane at points A and $\mathrm{B}, \mathrm{YZ}$ plane at point $\mathrm{E}, \mathrm{Y}$ direction at points $\mathrm{F}, \mathrm{YZ}$ plane at point $\mathrm{H}$ and finally back to $\mathrm{XZ}$ plane at point $\mathrm{J}$. In addition, Table 3 displays the nonlinear connections that are active at the considered points together
Fig. 15 3:1 interaction between NNM6 and NNM17. a Close-up of the internal resonance branch; $\mathbf{b}$ motion of the center of gravity of the WEMS cross

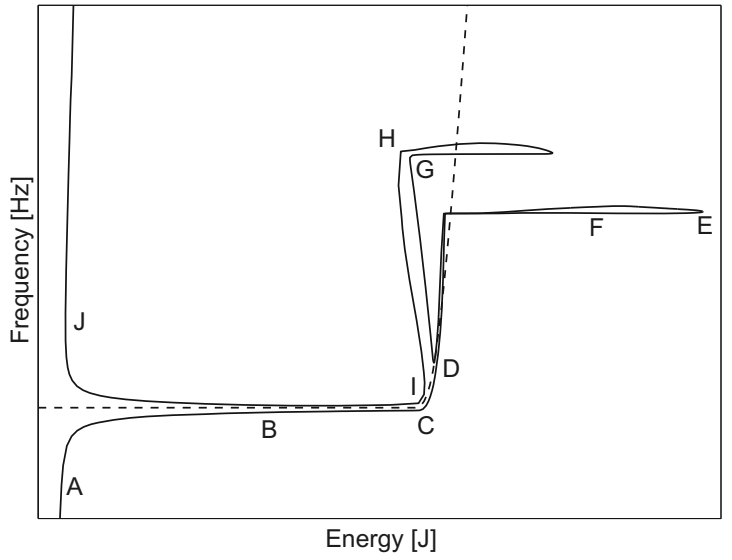

(a)
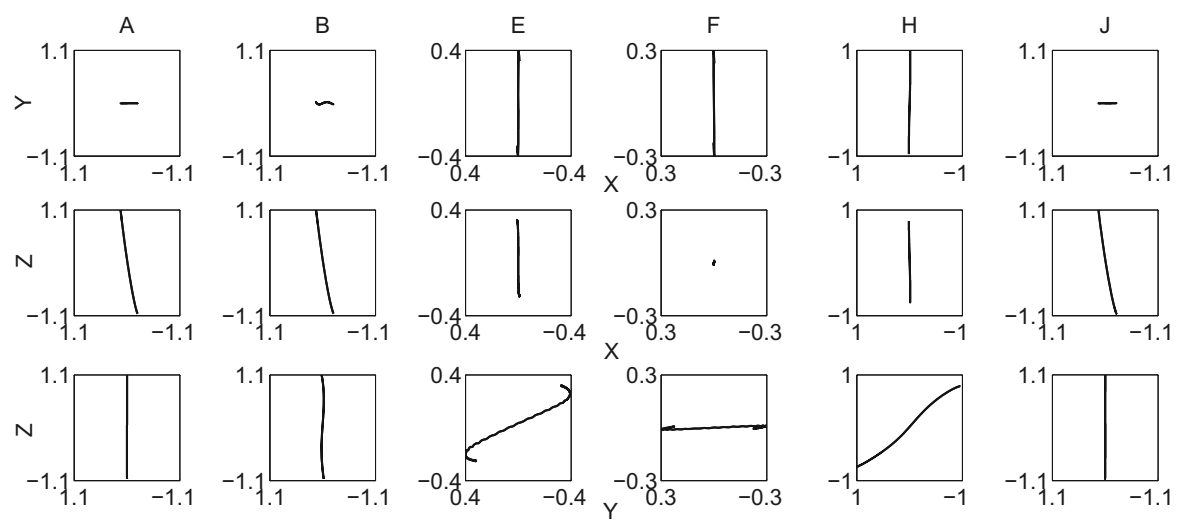

(b) 
Fig. 16 Influence of the size of the regularization interval on the FEP of NNM6. The nominal interval considered throughout the paper is equal to $5 \%$ of the clearance size black lines; blue lines $1 \%$, and red lines $10 \%$. (Color figure online)

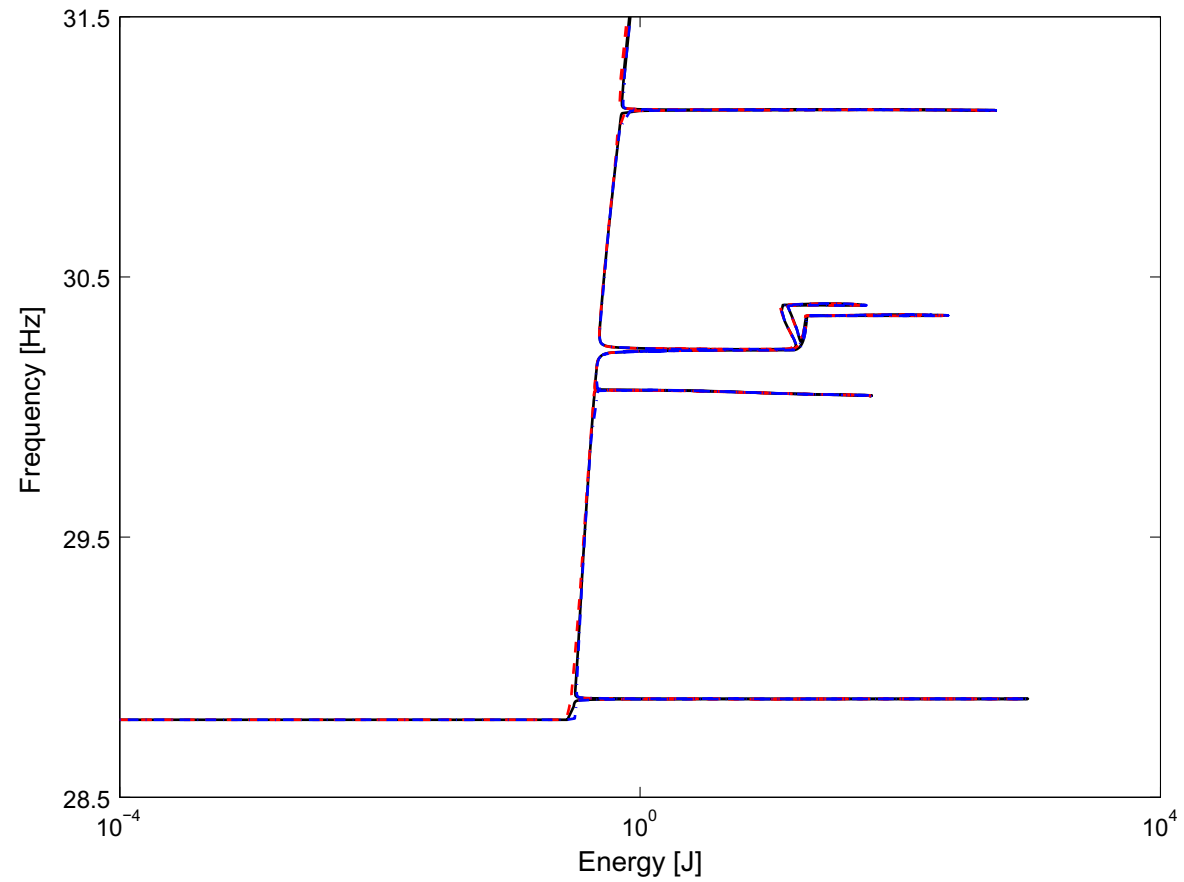

with the penetration in the corresponding regularization intervals.

At point A on the backbone of NNM6, the WEMS and the bracket vibrates axially resulting in activation of NC2-Z for negative relative displacements. At point $\mathrm{B}$ on the internal resonance branch, the WEMS motion is not significantly affected, but lateral motion of the bracket in the $\mathrm{Y}$ direction is induced. The MAC between NNM6-B and LNM6 is 0.5, a clear sign of the departure from mode 6 . At point $\mathrm{C}$, the MAC between NNM6-C and LNM17 is 0.92 indicating that the transition to NNM17 is well initiated. A second axial connection $(\mathrm{NC} 3-\mathrm{Z})$ is activated for negative relative displacements, but there is no visible frequency increase between points $\mathrm{B}$ and $\mathrm{C}$. As indicated in Table 3, the reason is that the motion barely penetrates in the regularization interval of NC3-Z. Conversely, a sudden frequency increase, which closely follows the evolution of the backbone of NNM17, is observed between points $\mathrm{C}$ and $\mathrm{D}$. This is a nonconventional behavior for an internal resonance branch. This occurs because the interaction with NNM17 generates lateral motion of the WEMS, which, in turn, activates two additional lateral connections, NC3-Y and NC4-Y. At point D, there are therefore 4 active connections. They have an important influence on the resulting dynamics, because the motion crosses the regularization zones for three of them. For a complete correspondence with NNM17, the two axial connections $\mathrm{NC} 2-\mathrm{Z}$ and $\mathrm{NC} 3-\mathrm{Z}$ must remain quiescent. This happens at point $\mathrm{E}$, but $\mathrm{NC} 4-\mathrm{Z}$ has been activated between points $\mathrm{D}$ and $\mathrm{E}$. It is only at point $\mathrm{F}$ that the axial connections are no longer active; the MAC with mode 17 is 0.99 . From point $\mathrm{F}$ to point $\mathrm{J}$, a reverse scenario for evolving from NNM17 back to NNM6 is observed in Table 3. Axial connections are again activated, and lateral connections become quiescent. We note that all these results hold for different sizes of the regularization intervals, as shown in Fig. 16.

Another interesting nonlinear phenomenon that the SmallSat satellite exhibits is the so-called localization phenomenon [18,33,34] during which new nonlinear modes with deformation localized to specific components of the structure appear. Figure 17 presents the FEP of NNM 7. Unlike previously described NNMs, NNM7 frequency remains identical to the linear natural frequency until energies greater than $10^{2} \mathrm{~J}$. Then, the backbone undergoes a bifurcation and bends backwards. Important modifications of the modal shapes are observed along the backbone branch. The linear-like modal shape only involves instrument panel motion. After the bifurcation, a markedly different $(\mathrm{MAC}<0.5$ ) modal shape involving significant WEMS motion is produced. Progressing on the backbone, the WEMS deformation is further enhanced, 
Fig. 17 FEP of the seventh NNM with different modal shapes inset

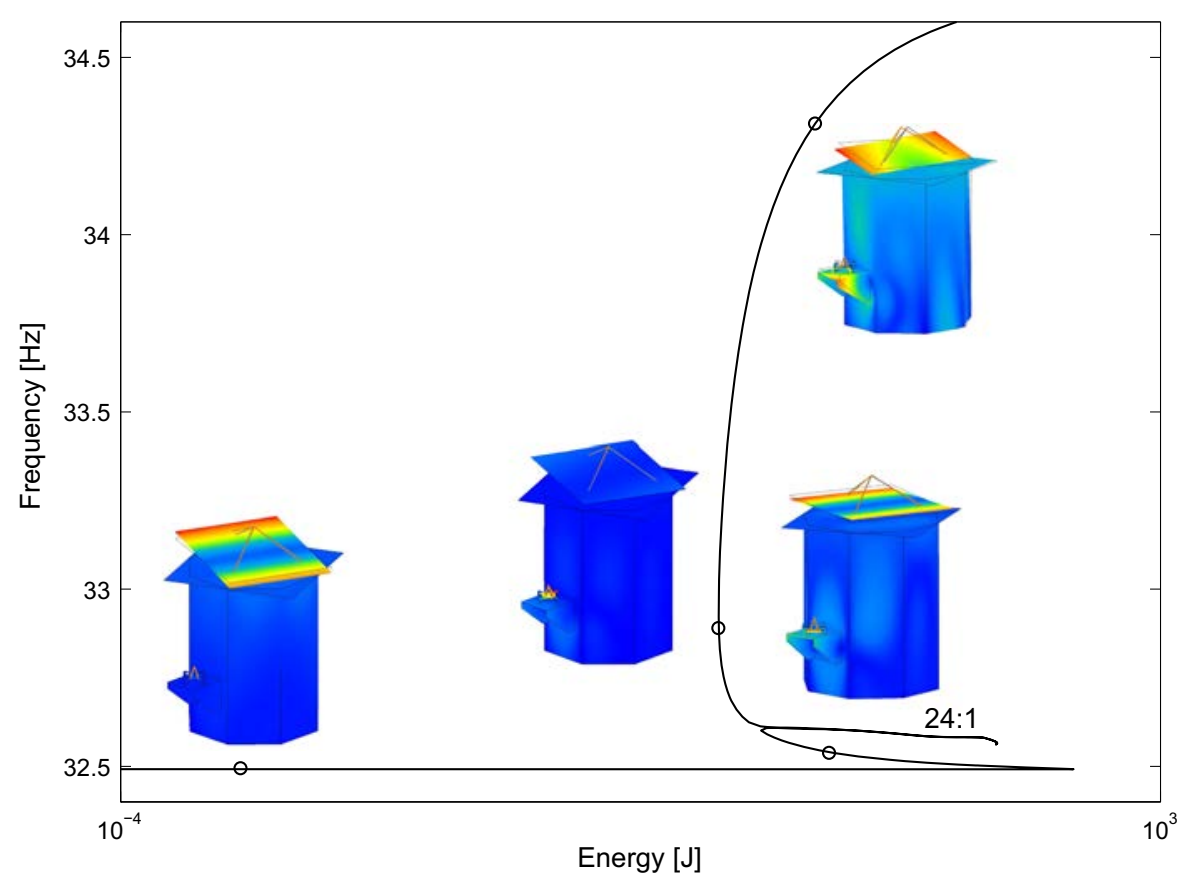

whereas instrument panel motion disappears, giving rise to a localized mode possessing some similarities with LNM6 (MAC $=0.85)$. Finally, a motion of the instrument panel, different from the motion at low energy, reappears further on the branch. We note that the localization phenomenon is different from modal interactions. For instance, very little harmonic components are generated along the backbone in Fig. 17.

Finally, the NNMs computed in this section are related to the forced continuation curves discussed in Sect. 4. The satellite response in the vicinity of the sixth resonance computed through forced numerical continuation is represented in solid line in Fig. 18. The external forcing is a stepped sine with different amplitudes, namely $20,55,60,75,80$ and $100 \mathrm{~N}$. In view of the sudden skewness of the resonance peaks, the nonsmooth character of the WEMS nonlinearities is evident. The frequency-energy dependence of NNM6 in Fig. 13 is also depicted in dashed line in Fig. 18. Clearly, the backbone branch computed in the undamped, unforced case coincides with the locus of the resonance peaks of the damped, forced response (marked with $*$ in Fig. 18). This confirms the well-known result that nonlinear resonances occur in the neighborhood of NNMs [18]. Unlike the backbone branch, the $2: 1$ modal interaction, represented by a vertical dashed line in Fig. 18,

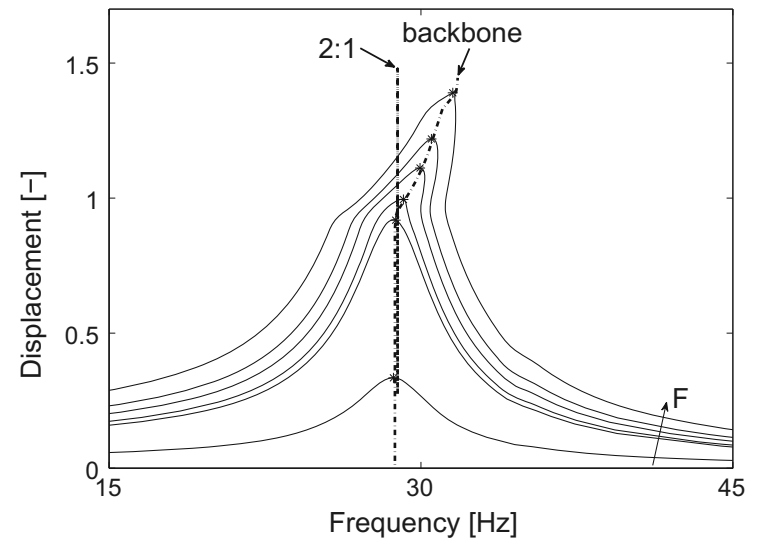

Fig. 18 Undamped/unforced (dashed line) versus damped/forced (solid line) response at the WEMS (NC4$\mathrm{Z}$ ) in the vicinity of the sixth resonance. Asterisk resonance locus in the damped/forced response

could not be reproduced in the forced continuation curves.

\section{Conclusions}

The objective of this paper was to investigate the dynamics of a real-life aerospace structure with a strongly nonlinear component. Due to the presence of multiple nonsmooth nonlinearities, closely spaced 
modes and relatively high damping, this application example poses several challenges. The advanced simulations carried out using numerical continuation showed that the satellite can exhibit a wide variety of nonlinear phenomena including jumps, rich frequency content, quasiperiodic motion, energy transfers from local to global structural modes, internal resonance branches with nonconventional topology, and mode localization. One specific contribution of this work is that several interactions between modes with noncommensurable linear frequencies, observed experimentally, were reproduced with great fidelity using numerical experiments. Overall, a very good qualitative agreement with the results of [15] was obtained. This demonstrates that there now exist in the technical literature effective and rigorous numerical and experimental methods for the analysis of complex, nonlinear industrial structures.

Finally, it is worth noting that the NNMs of the conservative system proved useful to interpret the modal interactions of the real structure. Future investigations should study the influence of damping on the results using, for instance, the concept of NNMs defined as two-dimensional invariant manifolds. Different tools for their computation were recently developed in $[35,36]$.

Acknowledgments This paper was prepared in the framework of the European Space Agency (ESA) Technology Research Programme study "Advancement of Mechanical Verification Methods for Nonlinear Spacecraft Structures (NOLISS)" (ESA contract No. 21359/08/NL/SFe). The authors also thank LMSSAMTECH for providing access to the SAMCEF finite element software. The authors L. Renson and J. P. Noël are Research Fellows (FRIA fellowship) of the Fonds de la Recherche Scientifique -FNRS, which is gratefully acknowledged.

\section{References}

1. Oueini, S.S., Nayfeh, A.H.: Analysis and application of a nonlinear vibration absorber. J. Vib. Control 6(7), 999-1016 (2000)

2. Vakakis, A.F., Gendelman, O., Bergman, L.A., McFarland, D.M., Kerschen, G., Lee, Y.S.: Nonlinear Targeted Energy Transfer in Mechanical and Structural Systems. Series: Solid Mechanics and Its Applications, vol. 156. Springer, Berlin (2009)

3. Gourdon, E., Alexander, N.A., Taylor, C.A., Lamarque, C.H., Pernot, S.: Nonlinear energy pumping under transient forcing with strongly nonlinear coupling: theoretical and experimental results. J. Sound Vib. 300(3-5), 522-551 (2007)
4. Carrella, A., Ewins, D.J.: Identifying and quantifying structural nonlinearities in engineering applications from measured frequency response functions. Mech. Syst. Signal Process. 25(3), 1011-1027 (2011)

5. Ahlquist, J.R., Carreno, J.M., Climent, H., Diego, R., Alba, J.: Assessment of nonlinear structural response in A400M GVT. In: Proceedings of the International Modal Analysis Conference, Jacksonville, USA (2011)

6. Fuellekrug, U., Goege, D.: Identification of weak nonlinearities within complex aerospace structures. Aerosp. Sci. Technol. 23(1), 53-62 (2012)

7. Platten, M.F., Wright, J.R., Cooper, J.E., Dimitriadis, G.: Identification of a nonlinear wing structure using an extended modal model. J. Aircr. 46(1), 1614-1626 (2009)

8. Noël, J.P., Renson, L., Kerschen, G., Peeters, B., Manzato, S., Debille, J.: Nonlinear dynamic analysis of an F-16 aircraft using GVT data. In: Proceedings of the International Forum on Aeroelasticity and Structural Dynamics, Bristol, UK (2013)

9. Carney, K.S., Yunis, I., Smith, K., Peng, C.Y.: Nonlinear dynamic behavior in the Cassini spacecraft modal survey. In: Proceedings of the International Modal Analysis Conference, Orlando, USA (1997)

10. Link, M., Boeswald, M., Laborde, S., Weiland, M., Calvi, A.: Non-linear experimental modal analysis and application to satellite vibration test data. In: Proceedings of the COMPDYN Conference, Corfu, Greece (2011)

11. Petrov, E.P., Ewins, D.J.: Advanced modeling of underplatform dampers for analysis of bladed disk vibration. J. Turbomach. 129, 143-150 (2007)

12. Laxalde, D., Thouverez, F., Sinou, J.J., Lombard, J.P.: Qualitative analysis of forced response of blisks with friction ring dampers. Eur. J. Mech. A/Solids 26, 676-687 (2007)

13. Wei, F., Liang, L., Zheng, G.T.: Parametric study for dynamics of spacecraft with local nonlinearities. AIAA J. 48, 17001707 (2010)

14. Knowles, J.A.C., Krauskopf, B., Lowenberg, M.H.: Numerical continuation applied to landing gear mechanism analysis. J. Aircr. 48, 1254-1262 (2011)

15. Noël, J.P., Renson, L., Kerschen, G.: Complex dynamics of a nonlinear aerospace structure: experimental identification and modal interactions. J. Sound Vib. 333(12), 2588-2607 (2014)

16. Muñoz-Almaraz, F.J., Freire, E., Galán, J., Doedel, E., Vanderbauwhede, A.: Continuation of periodic orbits in conservative and hamiltonian systems. Phys. D Nonlinear Phenom. 181(1-2), 1-38 (2003)

17. Dankowicz, H., Schilder, F.: Recipes for Continuation. Computational Science and Engineering, vol. 11, p. 579. SIAM (2013). doi:10.1137/1.9781611972573

18. Vakakis, A.F., Manevitch, L.I., Mlkhlin, Y.V., Pilipchuk, V.N., Zevin, A.A.: Normal Modes and Localization in Nonlinear Systems. Wiley-VCH Verlag GmbH, Weinheim (2008)

19. Shaw, S.W., Pierre, C.: Normal modes for non-linear vibratory systems. J. Sound Vib. 164, 40 (1993)

20. Russell, A.G.: Thick skin, faceted, CFRP, monocoque tube structure for smallsats. In: European Conference on Spacecraft Structures, Materials and Mechanical Testing, Noordwijk, The Netherlands (2000) 
21. Camarasa, P., Kiryenko, S.: Shock attenuation system for spacecraft and adaptor (SASSA). In: European Conference on Spacecraft Structures, Materials and Mechanical Testing, Toulouse, France (2009)

22. Bampton, M.C.C., Craig Jr, R.R.: Coupling of substructures for dynamic analyses. AIAA J. 6(7), 1313-1319 (1968)

23. Masri, S.F., Caughey, T.K.: A nonparametric identification technique for nonlinear dynamic problems. J. Appl. Mech. 46(2), 433-447 (1979)

24. Renson, L., Kerschen, G., Newerla, A.: Nonlinear modal analysis of the SmallSat spacecraft. In: Proceedings of the International Modal Analysis Conference, Orlando, USA (2012)

25. Renson, L., Noël, J.-P., Kerschen, G., Newerla, A.: Nonlinear modal analysis of the SmallSat spacecraft. In: Proceedings of the European Conference on Spacecraft Structures, Materials and Environmental Testing, Noordwijk, The Netherlands (2012)

26. Seydel, R.: Practical Bifurcation and Stability Analysis, Interdisciplinary Applied Mathematics, vol. 5. Springer, New York (2010)

27. Kuznetsov, Y.A.: Elements of Applied Bifurcation Theory, Applied Mathematical Sciences, vol. 112. Springer, New York (2004)

28. Kerschen, G., Peeters, M., Golinval, J.C., Vakakis, A.F.: Nonlinear normal modes, part I: a useful framework for the structural dynamicist. Mech. Syst. Signal Process. 23(1), 170-194 (2009)

29. Peeters, M., Viguié, R., Sérandour, G., Kerschen, G., Golinval, J.C.: Nonlinear normal modes, part II: toward a practical computation using numerical continuation techniques. Mech. Syst. Signal Process. 23(1), 195-216 (2009)
30. Kerschen, G., Peeters, M., Golinval, J.C., Stéphan, C.: Nonlinear modal analysis of a full-scale aircraft, J. Aircr. 50(5), 1409-1419 (2013)

31. Lee, Y.S., Kerschen, G., Vakakis, A.F., Panagopoulos, P., Bergman, L., McFarland, D.M.: Complicated dynamics of a linear oscillator with a light, essentially nonlinear attachment. Physica D 204(1-2), 41-69 (2005)

32. Lee, Y.S., Nucera, F., Vakakis, A.F., McFarland, D.M., Bergman, L.A.: Periodic orbits, damped transitions and targeted energy transfers in oscillators with vibro-impact attachments. Physica D 238(18), 1868-1896 (2009)

33. Vakakis, A.F.: Non-linear normal modes (NNMs) and their applications in vibration theory: an overview. Mech. Syst. Signal Process. 11(1), 3-22 (1997)

34. Georgiades, F., Peeters, M., Kerschen, G., Golinval, J.C., Ruzzene, M.: Modal analysis of a nonlinear periodic structure with cyclic symmetry. AIAA J. 47(4), 1014-1025 (2009)

35. Laxalde, D., Thouverez, F.: Complex non-linear modal analysis for mechanical systems: application to turbomachinery bladings with friction interfaces. J. Sound Vib. 322(4-5), 1009-1025 (2009)

36. Renson, L., Deliége, G., Kerschen, G.: An effective finiteelement-based method for the computation of nonlinear normal modes of nonconservative systems. Meccanica (2014) 